\begin{tabular}{|c|c|}
\hline Title & Synthesis of Cauer-Equivalent Circuit Based on Model Order Reduction Considering Nonlinear Magnetic Property \\
\hline Author(s) & Sato, Y uki; Shimotani, Toshihito; Igarashi, Hajime \\
\hline Citation & $\begin{array}{l}\text { IEEE Transactions on Magnetics, 53(6), 1-4 } \\
\text { https://doi.org/10.1109/T MA G.2017.2684242 }\end{array}$ \\
\hline Issue Date & 2017-03-17 \\
\hline Doc URL & http:/hdl.handle.net/2115/73865 \\
\hline Rights & $\begin{array}{l}\text { (C) } 2015 \text { IEEE. Personal use of this material is permitted. Permission from IEEE must be obtained for all other uses, in } \\
\text { any current or future media, including reprinting/republishing this material for advertising or promotional purposes, } \\
\text { creating new collective works, for resale or redistribution to servers or lists, or reuse of any copy righted component of } \\
\text { this work in other works. }\end{array}$ \\
\hline Type & article (author version) \\
\hline File Information & Cauer_PVL_full_pdf.pdf \\
\hline
\end{tabular}

Instructions for use 


\title{
Synthesis of Cauer-Equivalent Circuit Based on Model Order Reduction Considering Nonlinear Magnetic Property
}

\author{
Yuki Sato ${ }^{1,2}$, Student Member, Toshihito Shimotani ${ }^{1}$, Hajime Igarashi ${ }^{1}$, IEEE Member \\ ${ }^{1}$ Graduate School of Information Science and Technology, Hokkaido University, 060-0814 Sapporo, Japan \\ ${ }^{2}$ Research Fellow of Japan Society for the Promotion of Science (JSPS), Tokyo, 102-0083, Japan
}

\begin{abstract}
The Cauer-equivalent circuit of electric apparatus is synthesized by applying the model order reduction (MOR) to the finite element equations. In this method, the admittance function of a given electric apparatus is expressed by a rational polynomial, from which the Cauer-equivalent circuit is directly synthesized. Magnetic saturation in the magnetic core is considered by introducing nonlinearity in the primal inductance of the circuit. The synthesized circuit is shown to express the input-output properties of inductors and induction heating devices in good accuracy.
\end{abstract}

Index Terms - Cauer circuit, model order reduction, Padé via Lanczos, finite element method.

\section{INTRODUCTION}

$\mathrm{E}$ LECTIRC APPARATUS such as inductor, transformers and motors are often expressed by means of equivalent circuits in the design of driving and control circuits. The conventional equivalent circuit modeling, however, have difficulties in treatment of magnetic nonlinearity, computation of eddy current and hysteresis losses, and expression of characteristics over a wide frequency range.

The Cauer-equivalent circuit of a steel sheet has been derived from the analytical solution to the quasi-static Maxwell equations [1]-[3]. The synthesized Cauer circuit does not only express the frequency property of the steel plate over wide frequency range in good accuracy, but also can treat saturated cores. The winding coils have also been modeled by the Cauer circuit on the basis of analytical approach [4] (see also references in [4]). These methods can be, however, applied only to electric apparatus with simple geometry which can be analyzed with analytical methods.

The authors have proposed the synthesis of the Fosterequivalent circuit from the finite element (FE) equation of a given electric apparatus based on the model order reduction (MOR) [5]-[8]. This method can synthesize the equivalent circuits of electric apparatuses with arbitrary geometry and also accurately express wide-range frequency characteristic provided that there is no magnetic nonlinearity.

In this study, we extend the above MOR-based method to consider magnetic saturation in the magnetic cores. The proposed method synthesizes the Cauer circuit from the rational polynomials derived from the quasi-static Maxwell equations using Padé approximation via the Lanczos processes (PVL) based-MOR [9]. We apply this method to synthesize the Cauerequivalent circuit of an inductor used in a DC-DC converter as

Manuscript received November 20, 2016; revised May 15, 2015 and June 1, 2015; accepted July 1, 2015. Date of publication July 10, 2015; date of current version July 31, 2015. (Dates will be inserted by IEEE; "published" is the date the accepted preprint is posted on IEEE Xplore ${ }^{\mathbb{R}}$; "current version" is the date the typeset version is posted on Xplore $®)$. Corresponding author: Y. Sato (e-mail: yukisato@em.ist.hokudai.ac.jp).

Digital Object Identifier (inserted by IEEE). well as an induction heating device to evaluate the validity and performance of the proposed method.

\section{FINITE ELEMENT METHOD}

Let us consider the Maxwell equations represented in the Laplace domain for quasi-static electromagnetic fields

$\operatorname{rot} \operatorname{rot} \boldsymbol{A}+s \sigma(\boldsymbol{A}+\operatorname{grad} \varphi)=i \sum_{k} \boldsymbol{j}_{0 k}$

$\operatorname{div}\{s \sigma(\boldsymbol{A}+\operatorname{grad} \varphi)\}=0$

where $\boldsymbol{A}, \varphi, v, \sigma, i$, and $\boldsymbol{j}_{0 k}$ are magnetic vector and scalar potentials, magnetic reluctivity, conductivity, current and unit current density, respectively. Applying the weighted residual method in conjunction with Gelerkin method to (1), we obtain

$$
\begin{aligned}
& \sum_{i} A_{i} \int_{\Omega} \operatorname{rot} N_{i} \cdot \operatorname{rot} N_{j} \mathrm{~d} \Omega+s \sigma \sum_{i} A_{i} \int_{\Omega} N_{i} \cdot N_{j} \mathrm{~d} \Omega \\
& \quad+s \sigma \sum_{k} \varphi_{k} \int_{\Omega} N_{i} \cdot \operatorname{grad} N_{k} \mathrm{~d} \Omega=i \sum_{k} \int_{\Omega} N_{i} \cdot j_{0 k} \mathrm{~d} \Omega
\end{aligned}
$$

where $\boldsymbol{N}_{i}$ and $N_{j}$ are the vector and the scalar interpolation functions, respectively. To determine the current $i$, we couple (2) with the circuit equation given by

$v_{i n}=\sum_{j}^{c} \Delta v_{j}+i \sum_{j}^{c} R_{j}$

where $v_{i n}, \Delta v_{j}$, and $R_{j}$ are the input voltage, voltage drop along the $j$-th coil and its resistance, respectively. Note that $\Delta v_{i}$ is a function of $\boldsymbol{A}$ and $\varphi$. By solving (2) and (3), we obtain the admittance of the equivalent circuit at any frequency. This computation can be effectively performed using MOR technique which will be described below. 


\section{SynthESIS OF CAUER-EQUiVAlENT CiRCUIT}

In the present method, we use PVL-based MOR [9] to compute the admittance function of a given apparatus, which is expressed by a rational polynomial function of frequency.

\section{A. $\quad P V L$-based MOR}

To formulate PVL-based MOR, we express (2) and (3) in the state equation as follows:

$\mathrm{K} \boldsymbol{x}+s \mathrm{~N} \boldsymbol{x}=\boldsymbol{b} v$

where $\mathrm{K}, \mathrm{N} \in R^{n \times n}, \boldsymbol{x}, \boldsymbol{b}, \boldsymbol{l} \in R^{n}, n$ is the degree of freedoms (DoFs) in (2) and (3). The output equation is

$i=l^{t} \boldsymbol{x}$

The transfer function for the system described by (5), which corresponds to the admittance function, is given by

$Y(s)=\boldsymbol{l}^{t}\left\{\mathbf{I}-\left(s-s_{0}\right) \mathrm{A}\right\}^{-1} \boldsymbol{r}$

where $\mathrm{A}=-\left(\mathrm{K}+s_{0} \mathrm{~N}\right)^{-1} \mathrm{~N}, \boldsymbol{r}=\left(\mathrm{K}+s_{0} \mathrm{~N}\right)^{-1} \boldsymbol{b}$, and $s_{0}$ is an expansion point. The eigenvalue decomposition of $\mathrm{A}$ results in

$$
\begin{aligned}
Y(s) & =\boldsymbol{l}^{t}\left\{\mathrm{I}-\left(s-s_{0}\right) \mathrm{S} \Lambda \mathrm{S}^{-1}\right\}^{-1} \boldsymbol{r}=\boldsymbol{f}^{t}\left\{\mathrm{I}-\left(s-s_{0}\right) \Lambda\right\}^{-1} \boldsymbol{g} \\
& =\sum_{i} \frac{f_{i} g_{i}}{1-\left(s-s_{0}\right) \lambda_{i}}
\end{aligned}
$$

where $S, \Lambda$ are the matrix composed of the eigenvectors and diagonal matrix composed of the eigenvalues and $\boldsymbol{f}=\mathrm{S}^{t} \boldsymbol{l}, \boldsymbol{g}=\mathrm{S}^{-1} \boldsymbol{r}$. The eigenvalue decomposition of A needs, however, long computational time. For this reason, we apply the Lanczos method to (6) to obtain

$$
Y(s)=\boldsymbol{l}^{t} \boldsymbol{r} \boldsymbol{e}_{1}^{t}\left\{\mathrm{I}-\left(s-s_{0}\right) \mathrm{T}_{q}\right\}^{-1} \boldsymbol{e}_{1}
$$

where $\boldsymbol{e}_{1}=[1,0,0, \ldots, 0]^{t} \in R^{q}, \mathrm{~T}_{q} \in R^{q \times q}$ is a tridiagonal matrix and $q$ is the number of iteration in the Lanczos process. When $q$ is be set much smaller than $n$, we can easily perform the eigenvalue decomposition of $\mathrm{T}_{q}$ to obtain

$$
Y(s)=\boldsymbol{l}^{t} \boldsymbol{r} \boldsymbol{e}_{1}^{t}\left\{\mathrm{I}-\left(s-s_{0}\right) \mathrm{S}_{q} \Lambda_{q} \mathrm{~S}_{\mathrm{q}}^{-1}\right\}^{-1} \boldsymbol{e}_{1}=\sum_{j=1}^{q} \frac{\boldsymbol{l}^{t} \boldsymbol{r} \mu_{j} v_{j}}{1-\left(s-s_{0}\right) \lambda_{j}}
$$

where $\mathrm{S}_{q}, \Lambda_{q}$ are the matrices including the eigenvectors and eigenvalues of $\mathrm{T}_{q}$ and $\boldsymbol{\mu}=\mathrm{S}_{q}{ }^{t} \boldsymbol{e}_{1}$ and $\boldsymbol{\nu}=\mathrm{S}_{q}^{-1} \boldsymbol{e}_{1}$. The admittance is now represented as a rational polynomial of $s$ in (9).

\section{B. $\quad$ Synthesis of Cauer-Equivalent Circuit}

The authors have proposed the synthesis method of the Foster-equivalent circuit shown in Fig. 1 via PVL-based MOR [5]. In this method, we can directly and uniquely synthesize the Foster circuit from (9). However, it would be difficult to consider the magnetic saturation in magnetic cores using the Foster circuit. For the Cauer circuit shown in Fig. 2, which is also called continued-fraction Cauer circuit [4], physical interpretation to the each section could be given; $R_{1}$ and $L_{1}$ correspond to the DC resistance of winding coils and the inductance at low frequencies, while $R_{j}$ and $L_{j}, j=2,3, \ldots$

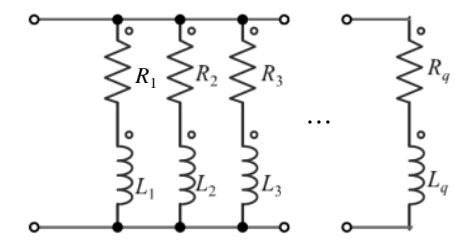

Fig. 1 Foster circuit.

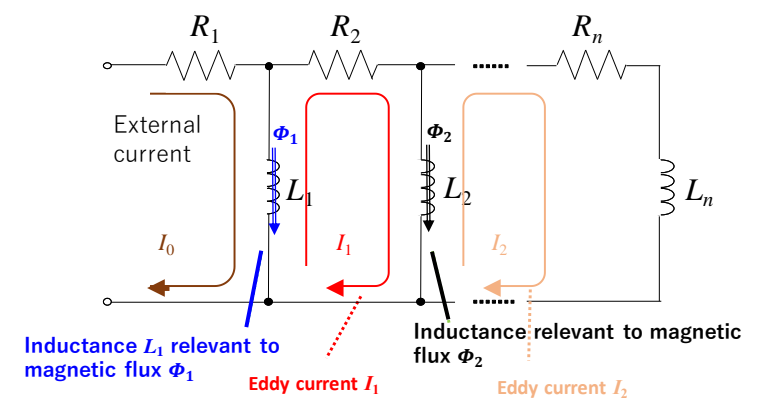

Fig. 2 Cauer circuit. The current $I_{0}$ denotes the external current while $I_{k}(k=1,2, \ldots)$ denote the eddy currents. The flux $\Phi_{1}$, generated by $I_{1}-I_{2}$, is dominant at low frequencies.

correspond to $\mathrm{AC}$ resistance and inductance relevant to the flux generated by the eddy currents. Because the flux $\Phi_{1}$ would be dominant, the magnetic saturation could be well described by introducing nonlinearity in $L_{1}[1]$. For this reason, we synthesize the Cauer-equivalent circuit from (9). To do so, we express (9) in the form

$Y(s)=\frac{b_{0}+b_{1} s+\cdots+b_{q-1} s^{q-1}}{a_{0}+a_{1} s+a_{2} s^{2}+\cdots+a_{q} s^{q}}$

in which the coefficients can be easily computed by, e.g., Mathematica ${ }^{\circledR}$. The Euclidian algorithm is applied to (10) to obtain the continued fraction

$$
Y(s)=\frac{1}{R_{1}+\frac{1}{1 / s L_{1}+\frac{1}{R_{2}+\frac{1}{1 / s L_{2}+\cdots}}}}
$$

where $R_{i}$ and $L_{i}$ are the resistance and inductance in the continued fraction Cauer circuit shown in Fig. 2. Note here that so-called physical Cauer circuit (see [4] for its definition) can also be synthesized from (10).

\section{Consideration of Nonlinearity in Magnetic Core}

As described above, we introduce the magnetic nonlinearity in magnetic cores only for $L_{1}$ [1]. When the eddy currents are dominant as in the case discussed in [10], we would have to introduce nonlinearity also in $L_{i}, i=2,3, \ldots$, as discussed in [3]. The circuit equation for the nonlinear Cauer is given by

$$
\begin{aligned}
v_{i n} & =\frac{\mathrm{d} \Phi\left(i_{1}-i_{2}\right)}{\mathrm{d} t}+R_{1} i_{1} \\
0 & =\frac{\mathrm{d} \Phi\left(i_{2}-i_{1}\right)}{\mathrm{d} t}+L_{2} \frac{\mathrm{d}\left(i_{2}-i_{3}\right)}{\mathrm{d} t}+R_{2} i_{2} \\
& \vdots \\
0 & =L_{q-1} \frac{\mathrm{d}\left(i_{q}-i_{q-1}\right)}{\mathrm{d} t}+L_{q} \frac{\mathrm{d} i_{q}}{\mathrm{~d} t}+R_{q} i_{q}
\end{aligned}
$$




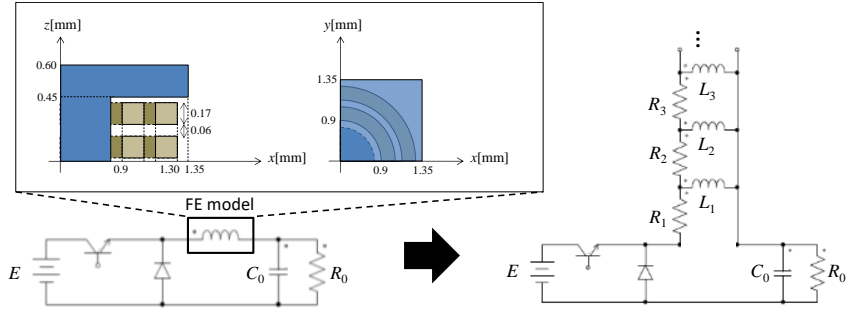

Fig. 3 Inductor in DC-DC converter.

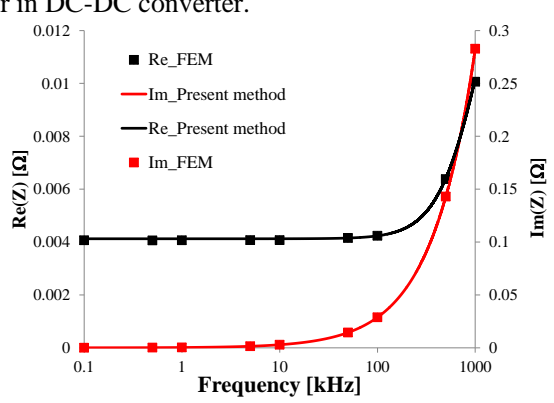

Fig. 4 Frequency response of impedance of the inductor

TABLE I ELEMENT VALUES OF INDUCTOR IN CAUER CIRCUIT.

\begin{tabular}{lllll}
\hline \hline$R_{1}[\Omega]$ & $R_{2}[\Omega]$ & $R_{3}[\Omega]$ & $R_{4}[\Omega]$ & $R_{5}[\Omega]$ \\
\hline \hline $3.30 \mathrm{e}-1$ & 59.2 & 968 & 2176 & 6328 \\
\hline \hline$L_{1}[\mathrm{H}]$ & $L_{2}[\mathrm{H}]$ & $L_{3}[\mathrm{H}]$ & $L_{4}[\mathrm{H}]$ & $L_{5}[\mathrm{H}]$ \\
\hline \hline $3.67 \mathrm{e}-7$ & $8.40 \mathrm{e}-6$ & $3.94 \mathrm{e}-5$ & $1.14 \mathrm{e}-4$ & $1.10 \mathrm{e}-4$ \\
\hline
\end{tabular}

where $i_{j}$ is the loop current in the $j$-th stage of the Cauer circuit. Instead of $L_{1} i$, we use $\Phi(i)$ to represent the nonlinearity of the magnetic core. Because $\Phi(i)$ is generated by the external coil current, $\Phi(i)$ can be determined by magneto- static field analysis. We compute the magnetic flux $\Phi(i)$ for different coil currents in the preprocessing.

The synthesis algorithm is summarized as follows:

1. The tri-diagonal matrix $T_{q}$ is generated by the Lanczos process. In this process, the eddy current equation (5a) is repeatedly solved.

2. The rational polynomial (10) is obtained by performing the eigenvalue decomposition of $T_{q}$, and the circuit parameters in the Cauer circuit are obtained by the continued fraction (11). 3. Magnetostatic field is analyzed with FEM to obtain $\Phi(i)$.

\section{NUMERICAL RESULTS}

\section{A. Inductor Model in DC-DC Converter}

We synthesize the Cauer-equivalent circuit of the inductor used in the DC-DC converter shown in Fig. 3. The conductivity of the coil is set to $5.76 \times 10^{7} \mathrm{~S} / \mathrm{m}$. The magnetic core is assumed to be 50A400. The inductor model is discretized into tetrahedral elements to whose edges 117,548 unknowns are assigned. In PVL-based MOR, we set the number of iteration $q$ to 5 which corresponds to the number of the stages in the Cauer circuit.

We summarize $R_{j}, L_{j}$ obtained by the present method assuming the linear magnetic property in Table I. The computed values of $R_{1}$ and $L_{1}$ correspond to the DC resistance and inductance at low frequency, respectively. The input impedance of the inductor is plotted against frequency in Fig. 4. We can see that the impedance computed from the Cauer circuit is in good agreement with that obtained by FEM.

Next, we analyze the DC-DC converter in which the inductor is modeled by the Cauer circuit. In this converter, $R_{0}$, switching

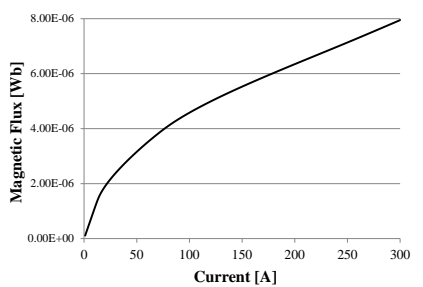

Fig. 5 Magnetic flux-current characteristic for inductor.

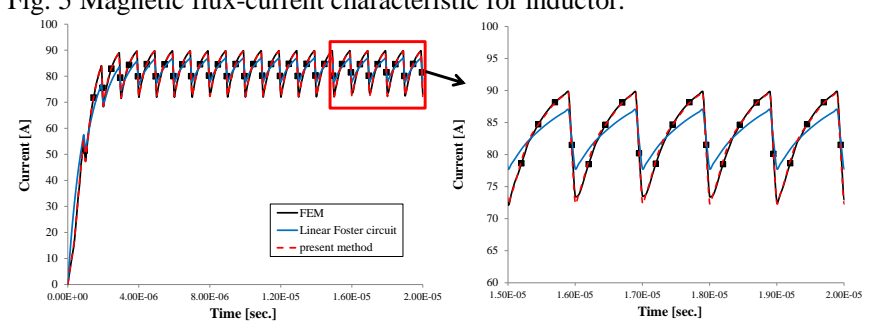

(a) $E=5.0 \mathrm{~V}, C_{0}=1 \mu \mathrm{F}$
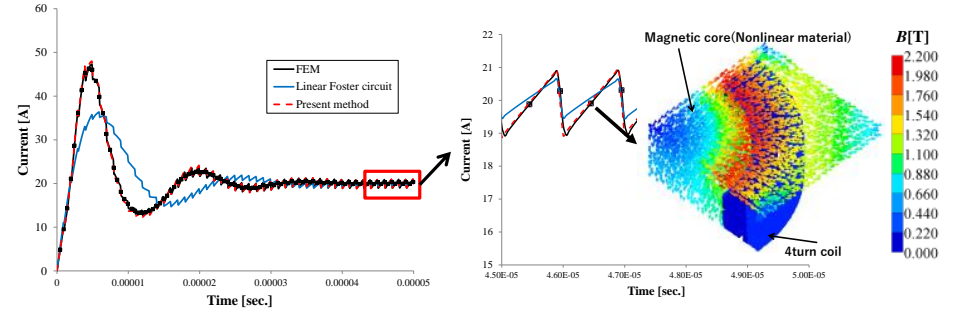

(b) $E=1.2 \mathrm{~V}, C_{0}=100 \mu \mathrm{F}$

Fig. 6 Time response of current which flows through $R_{0}$.

frequency and the duty factor are set to $0.05 \Omega, 1 \mathrm{MHz}$ and 0.9 , respectively. The nonlinear $\Phi-i$ characteristic of the core material, which is computed from the BH curve of 50A400, shown in Fig. 5 is introduced to $L_{1}$. We analyze the timeresponse using conventional FEM, present method and linear Foster-equivalent circuit which is synthesized using the permeability distribution at the driving current [2]. When $E=5.0 \mathrm{~V}, C_{0}=1 \mu \mathrm{F}$ and $E=1.2 \mathrm{~V}, C_{0}=100 \mu \mathrm{F}$, the time response of the current through $R_{0}$ is shown in Fig. 6. It is shown in Fig. 6 that the time responses obtained by FEM and the present method agree well. On the other hand, there are differences between those and the results computed from the Foster circuit. In particular, the differences are rather large in the transients.

\section{B. Induction Heating Model}

We consider the induction heating model shown in Fig. 7. The conductivity of the conducting plate to be heated is set to $1.25 \times 10^{6} \mathrm{~S} / \mathrm{m}$. The magnetic core is again assumed to be composed of 50A400. The induction heating model is discretized into tetrahedral elements which have 692,797 unknowns at the edges. In PVL-based MOR, $q$ is set to 5 .

In Table II, the values of $R_{j}$ and $L_{j}$ resulted from the present method assuming the linear magnetic property are summarized. The computed values of $R_{1}$ and $L_{1}$ are found to coincide with the DC resistance and inductance computed by static analysis, respectively. In the Cauer circuit of the induction heating model, the higher element $R_{j}$ and $L_{j}, j=2,3, \ldots$ correspond to the resistance and the inductance relevant to the eddy currents in the metallic plate. Figure 8 shows the frequency dependence of the input impedance of the coil obtained by FEM and the Cauer circuit. They are in good agreement. 


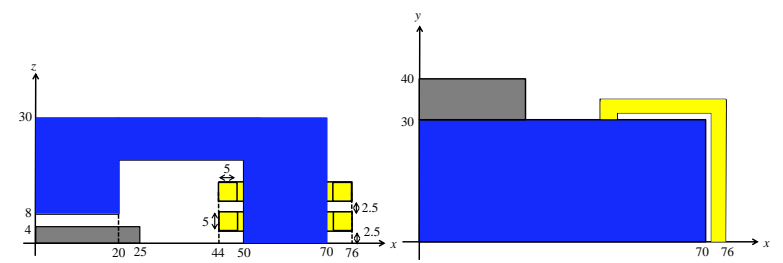

Fig. 7 Induction heating model (blue : magnetic core, yellow : coil, gray : conducting plate)

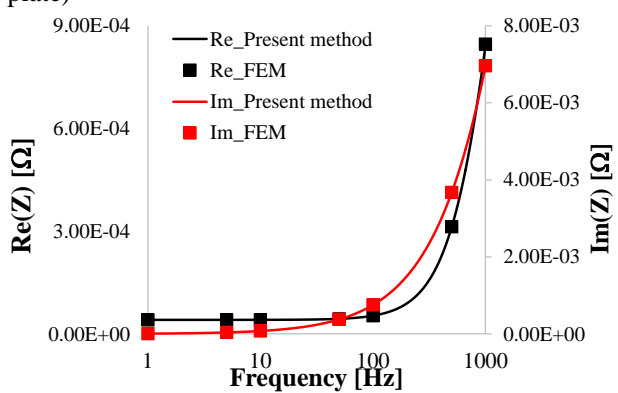

Fig. 8 Frequency response of impedance of the induction heating TABLE II Element values of Induction Heating in Cauer circuit.

\begin{tabular}{lllll}
\hline \hline$R_{1}[\Omega]$ & $R_{2}[\Omega]$ & $R_{3}[\Omega]$ & $R_{4}[\Omega]$ & $R_{5}[\Omega]$ \\
\hline \hline $3.28 \mathrm{e}-4$ & $3.69 \mathrm{e}-1$ & 10.1 & 71.8 & 54.0 \\
\hline \hline$L_{1}[\mathrm{H}]$ & $L_{2}[\mathrm{H}]$ & $L_{3}[\mathrm{H}]$ & $L_{4}[\mathrm{H}]$ & $L_{5}[\mathrm{H}]$ \\
\hline \hline $9.60 \mathrm{e}-6$ & $3.32 \mathrm{e}-5$ & $2.21 \mathrm{e}-4$ & $9.36 \mathrm{e}-4$ & $1.19 \mathrm{e}-3$ \\
\hline
\end{tabular}

We analyze the induction heating model in time-domain considering the magnetic nonlinearity in the magnetic core. By performing the magneto-static field analysis, we compute $\Phi-i$ characteristic of the core, which is plotted in Fig. 9. We introduce the nonlinear characteristic to $L_{1}$ in the Cauer circuit. In Fig. 10, we plot the time variation of the current to the coil winding. The solutions in the both transient and steady states obtained by FEM agree well with those by the nonlinear Cauer circuit even when the voltage $E$ changes, although the linear circuit has errors in the transients.

The Joule losses in the steady state evaluated by FEM and the Cauer circuit are shown in Fig. 11. From the Cauer circuit, we compute the Joule loss as follows:

$$
W_{e}=\sum_{j=2}^{q} R_{j} i_{r j}^{2}
$$

where $i_{r j}$ is the current to $R_{j}$. As can be seen in Fig. 11 , the Joule loss obtained by the Cauer circuit is in good agreement with that obtained by FEM.

\section{CONCLUSION}

We have proposed a novel method to synthesize the Cauer circuit from the FEM model of electric apparatus using PVLbased MOR. The synthesized Cauer circuit can express the nonlinear magnetic property of magnetic cores. It is shown that the Cauer circuit provides accurate results in both frequency and time domains. We plan to study the property of the physical Cauer circuit which is synthesized from (10).

\section{REFERENCES}

[1] E. J. Tarasiewicz, A. S. Morched, A. Narang, E. P. Dick, "Frequency Dependent Eddy Current Models for Nonlinear Iron Cores," IEEE Trans. on Power Systems, vol.8 no.2 pp. 588-597, May, 1993.

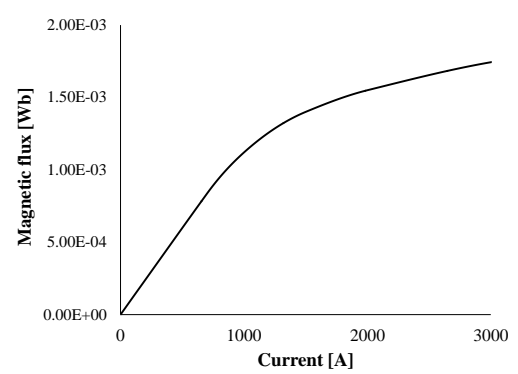

Fig. 9 Magnetic flux-current characteristic for induction heating.

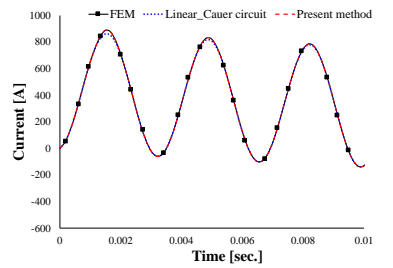

(a) Transient state when $E=1.0 \mathrm{~V}$

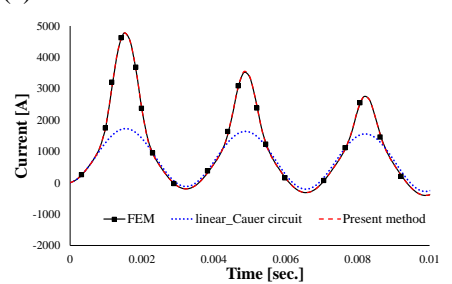

(c) Transient state when $E=2.0 \mathrm{~V}$

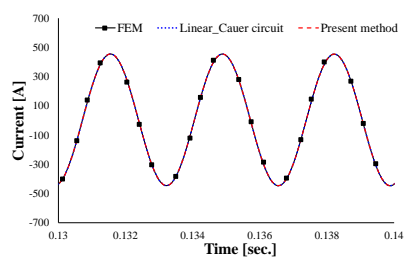

(b) Steady state when $E=1.0 \mathrm{~V}$

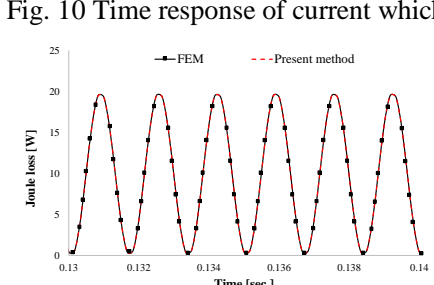

(a) $E=1.0 \mathrm{~V}$

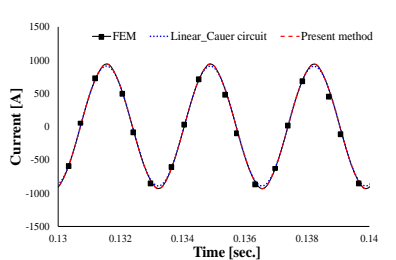

(d) Steady state when $E=2.0 \mathrm{~V}$
Fig. 10 Time response of current which flows through $R_{0}$.

Fig. 11 Time response of Joule loss in the magnetic steel.

[2] Y. Shindo, O. Noro, "Simple Circuit Simulation Models for Eddy Current in Magnetic Sheets and Wires," IEEJ Trans. Fundamentals and Materials, vol. 134, no. 4, pp. 173-181, 2014.

[3] T. Miyazaki, T. Mifune, T. Matsuo, Y. Shindo, Y. Takahashi, K. Fujiwara, "Equivalent Circuit Modeling of Dynamic Hysteretic Property of Silicon Steel under Pulse Width Modulation Excitation," Journal.of Applied Physics, 117, 17D110, 2015.

[4] S. Jazebi, F. de Leon, B. Vahidi, "Duality-Synthesized Circuit for Eddy Current Effects in Transformer Windings," IEEE Trans. Pow. Deli., vol. 28, no. 2, Apr., 1063-1072, 2013.

[5] Y. Sato, H. Igarashi, "Generation of Equivalent Circuit from Finite Element Model Using Model Order Reduction," IEEE Transaction on Magnetics, vol. 52, no. 3, Art. 1100304, 2016

[6] T. Shimotani, Y. Sato, H. Igarashi, "Equivalent-Circuit Generation from Finite Element Solution Using Proper Orthogonal Decomposition," IEEE Transaction on Magnetics, vol. 52, no. 3, Art. 7206804, 2016.

[7] Y. Sato, T. Mori, T. Shimotani, H. Igarashi, "Equivalent Circuit of Antennas Generated by Model Order Reduction," International Journal of Applied Electromagnetics and Mechanics, to be published.

[8] T. Shimotani, Y. Sato, H. Igarashi, "Direct Synthesis of Equivalent Circuits from Reduced FE Models Using Proper Orthogonal Decomposition," The International Journal for Computation and Mathematics in Electrical and Electronic Engineering(COMPEL) vol. 35, no. 6, 2016.

[9] P. Feldmann and R. A. Freund, "Efficient linear circuit analysis by Padé approximation via the Lanczos process," IEEE Trans. Computer-Aided Design, vol. 14, no. 5, pp. 639-649, May 1995.

[10] A. Elsherbini, H. Braunisch, K. O’Brien, "Circuit Modeling of Nonlinear Lossy Frequency-Dependent Thin-Film Magnetic Inductors," in IEEE Trans. Components, Packaging and Manufacturing Tech., vol. 4, no. 1, pp. 145-152, Jan. 2014. 